\title{
Valoración económica ambiental del recurso hídrico, y el beneficio para los usuarios del sistema de riego Guargualla de la parroquia Licto, cantón Riobamba provincia de Chimborazo (2012-2016)
}

Zurita Moreano, Eduardo

Universidad Nacional de Chimborazo, Ecuador Universidad Nacional de Chimborazo, Ecuador ezurita@unach.edu.ec

Lema Espinoza, Magdala de Jesús

Universidad Nacional de Chimborazo, Ecuador mlema@unach.edu.ec
Resumen - La presente investigación se centra en los afluentes del rio Guargualla que abastece el sistema de riego que lleva el mismo nombre, este se ubica en la parroquia Licto, cantón Riobamba, provincia de Chimborazo, siendo la única fuente de abastecimiento de agua para riego, se hizo necesario una valoración económica y ambiental, con el propósito de implementar un sistema de protección y cuidado de los afluentes. Esto contribuirá en mantener la cantidad y calidad del recurso hídrico para promover la producción agropecuaria de sus 1926 usuarios conformados por cuatro parroquias y 22 comités comunales de riego. El trabajo parte de un diagnóstico y evaluación del servicio ambiental hídrico del lugar, incluyendo dos aspectos importantes, la oferta y demanda del agua, para luego determinar el valor de captación y restauración, herramientas que per- mitirán identificar los beneficios que ocasiona la valoración del agua. Se utilizó el método de valoración contingente que sirve para estimar el valor de bienes y servicios que no existen en el mercado, es una valoración simple y de comprensión intuitiva, trata de suponer un mercado mediante la realización de encuestas a los consumidores potenciales. El estudio estableció que el $82 \%$ de los usuarios están conscientes de la disminución del recurso hídrico, por lo que el $91 \%$ están dispuestos a contribuir con un pago adicional para la conservación de los páramos. Se estableció el valor de 0,31 centavos de dólar para financiar la conservación de las fuentes del sistema de riego de la Junta Guargualla.

Palabras clave: recurso hídrico; sistema de riego; valoración contingente; valoración económica ambiental.
Interconectando Saberes, 2019

ISSN: 2448-8704
Fecha de Recepción: 10 de agosto de 2019

Fecha de Aceptación: 25 de septiembre de 2019

Fecha de Publicación: 31 de octubre de 2019 
Abstract - The present investigation focuses on the tributaries of the Guargualla river that supplies the irrigation system that bears the same name, it is located in the Licto parish, canton Riobamba, province of Chimborazo, being the only source of water supply for irrigation, It made necessary an economic and environmental assessment, with the purpose of implementing a system of protection and care of the tributaries. This will contribute to maintaining the quantity and quality of the water resource to promote the agricultural production of its 1926 users made up of four parishes and 22 communal irrigation committees. The work is based on a diagnosis and evaluation of the water environmental service of the place, including two important aspects, the supply and demand of water, to then determine the value of catchment and restoration, tools that will allow to identify the benefits that the valuation of water causes. We used the contingent valuation method that is used to estimate the value of goods and services that do not exist in the market, it is a simple and intuitive understanding, which is to assume a market by conducting surveys to potential consumers. The study established that $82 \%$ of users are aware of the decrease in water resources, so $91 \%$ are willing to contribute with an additional payment for the conservation of the páramos. The value of 0.31 cents was established to finance the conservation of the sources of the irrigation system of the Guargualla Board.

Keywords: hidric resource; irrigation system; contingent valuation; environmental economic valuation.

\section{INTRODUCCIÓN}

La valoración económica ambiental es un tema relativamente nuevo, debido a que los recursos naturales están agotándose en el planeta, el tema de la conservación y protección de los ecosistemas ha tomado gran importancia en la actualidad. En ese sentido, la conservación del recurso hídrico es fundamental para el fortalecimiento del desarrollo de una nación en el ámbito productivo y socio económico.

\section{Ecuador se caracteriza por tener} una gran riqueza natural, identificándolo como un país mega diverso, ya que concentra una gran variedad de fuentes hidrográficas. En la actualidad el recurso hídrico se ha visto favorecido por políticas de gobierno que han tratado de distribuir equitativamente el agua, para los diversos sectores productivos como: el sector agropecuario, industria, turístico, sin embargo, se ha avanzado muy poco, por lo que crear leyes es de trascendental importancia.

La presente investigación hace referencia a los afluentes del rio Guargualla del sistema de riego del mismo nombre de la parroquia Lic- 
to, cantón Riobamba, el cual se constituye en la única fuente de abastecimiento de agua para riego, por lo que es necesario una valoración económica y ambiental ya que sus beneficiarios se dedican a actividades como la agricultura y la ganadería, siendo la principal fuente de ingreso de las familias de la zona.

La investigación parte de un diagnóstico y evaluación del servicio ambiental hídrico del lugar, incluyendo dos aspectos importantes, la oferta y la demanda del agua, para luego determinar el valor de captación y restauración, herramientas que permitirán identificar los beneficios que ocasiona la valoración del agua para destinar un método de conservación.

Mediante dicho análisis se podrá determinar la importancia que tiene el recurso hídrico en el proceso productivo, para posteriormente plantear un sistema de cuidado y protección de los páramos donde nacen las vertientes del rio Guargualla, desde este punto de vista nace la necesidad de conservar los páramos para mantener la cantidad y calidad del recurso.
La investigación se desarrolló bajo el método de valoración contingente propuesta por Gerardo Barrantes Moreno, en el que propone que para realizar una valoración de un bien natural se debe simular un mercado mediante la aplicación de las encuestas, en donde se identifica la disposición de pago por parte de los beneficiarios del recurso natural.

\section{MATERIALES Y MÉTOdos}

Según Herruzo (2002), menciona que la valoración económica es un instrumento al servicio de la política ambiental mediante el cual se pretende imputar valores a los bienes. La valoración económica resulta necesaria para lograr dos objetivos económicos prioritarios en todo sistema económico: la eficiencia económica y el crecimiento sostenible.

Por su parte, Tomasini (2018) afirma que:

Valorar económicamente al ambiente supone el intento de asignar valores cuantitativos a los bienes y servicios proporcionados por los recursos ambientales, independientemente de la existencia de precios de mercado 
para los mismos. Esto quiere decir que la necesidad de la valoración excede largamente al trabajo que hace el mercado otorgando precios y asignando recursos dentro de la economía. Hay una enorme cantidad de bienes y servicios ambientales para los cuales es imposible encontrar un mercado donde se generen los "precios" que racionen su uso dentro del sistema. La valoración nos señala que el ambiente no es gratis, el desafio es expresar en términos. (pág.1)

\section{VALORACIÓN ECONÓMICA DEL MEDIO AMBIENTE}

De acuerdo a Romero (2014), la valoración económica del medio ambiente pretende cuantificar la disposición social a pagar, esta disposición cuenta con ciertos factores, como las características de los grupos de personas afectadas y el espacio temporal, que se reflejan en términos monetarios sobre las preferencias individuales por los bienes ambientales o la disposición de la perdida de la calidad ambiental.

Azqueta (2002) señala que "la valoración económica del medio ambiente es el poder de contar con un indicador del bienestar de la sociedad que permita compararlo con otros componentes del mismo" (pág. 15).

En ese sentido la (Organización de las Naciones Unidas, 2016) explica que por mucho tiempo el recurso hídrico (agua), se le ha tomado sólo como un factor productivo, sin tomar en cuenta el efecto sobre los demás recursos del ecosistema, en la actualidad, el papel que cumple el agua según los economistas es la relación que existe entre el agua, la riqueza y la economía.

\section{MÉTODO DE VALORACIÓN CONTINGENTE}

La valoración contingente (VC), está incluida en el método directo que se emplea para estimar el valor de bienes y servicios que no existen en el mercado, es una valoración simple y de comprensión intuitiva, lo que trata es de suponer un mercado mediante la realización de encuestas a los consumidores potenciales. De esta forma, se les preguntará cuanto estaría dispuesto a pagar por una máxima cantidad (disposición a pagar DAP), si tuvie- 
ra que adquirirlo, de esta manera se deduce el valor por parte del consumidor. El método de VC puede considerar la pérdida de un bien por medio de la disposición a ser compensado (DAC) (Riera, 1994). Por lo que es necesario delimitar la extensión y las características del bien a valorar. Se debe interrogar sobre el DAP o la DAC.

\section{DESARROLLO DE LOS}

\section{PARÁMETROS BÁSICOS DE ESTUDIO}

En lo referente a los parámetros básicos Riera (1994) explica que:

- Se debe conocer la población objetivo.

- Seleccionar la muestra, se debe ajustar las variables necesarias y expresarse mediante el nivel de confianza y el margen de error.

- Se debe decidir qué tipo de medida de bienestar se va a valorar y estimar, sea esta la variación compensatoria o la variación equivalente.

En el caso de variación compensatoria $(\mathrm{V} / \mathrm{c})$ es un escenario donde se pierde la calidad y cantidad del bien público, se la expresa de la siguiente manera:

$$
U\left(p, y, r^{\circ}\right)=U\left(p, y+V c, r^{1}\right)
$$

\section{Donde:}

$U=$ Utilidad

$p=$ Función de los precios

$y=$ Nivel de renta

$r=$ Cantidad y calidad ambiental recreativo (0) inicial, (1) final.

$V c=$ Variación compensatoria .

Para explicar lo anteriormente mencionado, (Cerda, 2009) establece que el consumidor es indiferente al consumir un bien público, a nivel inicial de calidad o cantidad y consumir menos de dicho bien si se incrementa su renta monetaria con la Vc, en otras palabras, Vc corresponde a la mínima cantidad de dinero que el consumidor debería recibir en compensación para sentirse indiferente ante la disminución de la cantidad o calidad de un bien. Así mismo, en el caso de mejorar las condiciones del ecosistema, el signo Vc tendría una expresión negativa, en este caso es cuánto dinero está dispuesto a pagar el consumidor por la mejora del sitio.

La ecuación de la variación equivalente $(\mathrm{Ve})$, que representa la utilidad es; 


$$
U\left(p, y, r^{\circ}\right)=U\left(p, y-V e, r^{1}\right)
$$

\section{Donde:}

$V e=$ máxima cantidad de dinero que tiene el consumidor dispuesto a pagar.

$r=$ En el caso contrario, con (Ve +) en la formula, es la mínima cantidad de dinero con la que debería compensar a un consumidor para que volviera a su utilidad original después de un daño al bien público.

\section{Aspectos METODOLÓGICOS}

En la presente investigación se utilizó el método lógico inductivo el cual permitió realizar un diagnóstico de la situación del recurso hídrico adjudicado al sistema de riego Guargualla Licto y conocer los beneficios y oportunidades que se ha generado en el sector productivo de forma cuantitativa. Aplicando la formula en la población general se obtiene una muestra de 320 encuestas.

Se utilizó la técnica de la observación, la misma que nos permitió estar en contacto con la realidad de los beneficiaros y así poder precisar los beneficios y oportunidades que ha generado el riego en el sistema Guargualla Licto. Como instrumento se utilizó el cuestionario el cual nos permitió la obtención de resultados cuantificables e importantes para la valoración de recurso hídrico, además de la demanda y oferta que ofrece el sistema de riego y las características socioeconómicas de los usuarios.

Para la valoración económica se desarrolló un balance hídrico, como una variable que determina la oferta y la demanda que el sistema de riego. Por lo que según la sentencia otorgada por la Secretaria Nacional del Agua (SENAGUA) en marzo del 2010, la Junta General de Usuarios del Sistema de Riego Guargualla Licto dispone de una adjudicación $1.3 \mathrm{~m}^{3}$ de agua por segundo. La organización es de carácter mixto, por lo que las responsabilidades son compartidas en el manejo y cuidado de las diferentes actividades, con el Consejo provincial de Chimborazo, por lo mismo cada uno cobra un porcentaje para cubrir los gastos de mantenimiento y cuidado del canal principal. 


\section{RESULTADOS}

Como se observa en la Tabla 1, el costo de oportunidad promedio por familia es de 1461,83 dólares por año; señalando que los beneficiarios actualmente desarrollan sus actividades económicas dentro del sistema de riego, esto indica la importancia que tiene el recurso hídrico como medio de ingresos para sustentar a la familia, por lo que desde el punto de vista económicosocial los usuarios están conscientes y por ende comprometidos a conservar y proteger los afluentes del recurso hídrico de la organización.

\section{a.) Valor de captación}

\section{$V C=\Sigma \infty$ iBiAbi /Oci}

\section{Dónde:}

$$
\begin{aligned}
& V C=\text { Valor de captación hídrica } \\
& \text { del sistema de riego Guargua- } \\
& \quad l l a \\
& B=\text { Costo de oportunidad } \\
& \left(\$^{*} a n ̃ o / H a\right)=\$ 1.461,83
\end{aligned}
$$

$A b=$ Área de los páramos del rio

Guargualla utilizada $(\mathrm{ha})=$ 2200 ha.
Oc $=$ Volumen de agua captada en la cuenca $(\mathrm{m} 3 / \mathrm{año})=1300$ $\mathrm{m} 3 / \mathrm{s}=40,996.800 \mathrm{~m} 3 / \mathrm{año}$ $\infty=$ Importancia de recurso hídrico $0<\infty<1=72 \%=0.72$

$$
\mathbf{V C}=\frac{(\infty * \mathbf{B} * \mathbf{A b})}{\mathbf{0 c}}
$$

$$
\begin{gathered}
\text { VC }=\frac{0.72 * 1461,83 * 2200}{40.996800} \\
\text { VC }=\frac{2315538,72}{40996800} \\
\text { VC }=0,0564809624 \text { dólares } / \mathrm{m}^{3}
\end{gathered}
$$

Aplicando la fórmula de Barrantes se obtiene un valor de capta-

\begin{tabular}{|c|c|c|c|c|c|}
\hline No. Encuestados & $\begin{array}{l}\text { Rangos de } \\
\text { ingresos }\end{array}$ & $\mathrm{Ha}$ & $\begin{array}{c}\text { Ingreso } \\
\text { mensual/ha }\end{array}$ & $\begin{array}{c}\text { Ingreso Promedio } \\
\text { mensual / Ha }\end{array}$ & $\begin{array}{l}\text { Ingreso Promedio } \\
\text { anual/ ha }\end{array}$ \\
\hline 114 & 100 & 1 & 100 & & \\
\hline 82 & 200 & 2 & 100 & & \\
\hline 92 & 375 & 3 & 125 & & \\
\hline 16 & 437 & 4 & 109.25 & & \\
\hline 8 & 650 & 5 & 130 & & \\
\hline 8 & 1,000 & 6 & 16.67 & & \\
\hline 320 & ---- & ---- & ---- & 121.82 & 100 \\
\hline
\end{tabular}
ción de 0,0564809624 dólares por m3, lo que significa que el beneficiario debe realizar un aporte económico para compensar a los dueños de los páramos por no continuar con el avance de la frontera agrícola.

Tabla 1. Ingresos agropecuarios de los usuarios

Fuente: Encuestas. Elaboración Propia de los Autores 
b.) Valor de restauración

$$
\text { VR }=\sum \sum \bar{o} \mathbf{i j} \mathbf{C i j} \frac{\text { Ari }}{\text { Oci }}
$$

\section{Donde:}

$V R=$ Valor de restauración del sistema de riego Guargualla $C=$ Costos para la actividad destinada a la restauración sistema de riego Guarguallá $\left(\${ }^{*}\right.$ ha/año $)=264$ dólares Ar = Área a restaurar en el sistema de riego Guargualla i (ha) $=60$ ha.

$\bar{O}=$ Fracción del costo $\mathrm{j}$ destinado a la restauración del sistema de riego Guargualla en función del recurso hídrico $=$ $72 \%=0.72$

$0=$ Volumen de agua captada en la cuenca $(\mathrm{m} 3 / \mathrm{año})=1300$ $\mathrm{m} 3 / \mathrm{s}=40.996800 \mathrm{~m} 3 /$ año.

$$
\begin{gathered}
\mathbf{V R}=(\overline{\mathbf{o}} * \mathbf{C} * \mathbf{A r}) / \mathbf{O c} \\
\mathrm{VR}=\frac{0.72 * 264 * 60}{40996800} \\
=\frac{11404.80}{40996800}=0,0002781876 \text { dólares } / \mathrm{m}^{3}
\end{gathered}
$$

El cuidado y protección del recurso hídrico, en el desarrollo de las actividades productivas de la zona es importante, por lo tanto, la res- tauración del sistema de riego Guargualla debe contener una programación de actividades en para su conservación.

Para calcular el valor de restauración, se ha tomado en cuenta aspectos como: el área que se va a restaurar, de acuerdo al avance de la frontera agrícola, que es de 60 ha, de acuerdo a fuentes secundarias; la fracción de costo corresponde al $72 \%$ según las autoridades competentes. Con estos antecedentes se procede a calcular el costo real de la agro forestación de las 60 ha que han sufrido deforestación en los páramos de los afluentes del rio Guargualla, según el Ministerio del Ambiente y Consejo Provincial de Chimborazo, las 60 hectáreas a intervenir tienen un costo de 15840 USD por lo que dividiendo para el área a forestar tiene un costo de 264 dólares por ha, determinándose un valor de restauración de 0,0002781876 centavos de dólar por $\mathrm{m}^{3}$ al año.

Según el sistema hidráulico de la organización una hectárea se riega en seis horas con 6 litros de agua por segundo. Luego de determinar los valores de captación y de res- 
Tabla 2. Valor de forestación y captación

\begin{tabular}{|c|c|c|c|c|}
\hline SECTOR & $\begin{array}{l}\text { Demanda anual* de } \\
\text { agua } \mathrm{m}^{3} / \text { año } \mathrm{Ha}\end{array}$ & $\begin{array}{c}\text { VALOR DE } \\
\text { CAPTACIÓN (dólares } \\
\qquad / \mathrm{m}^{3}\end{array}$ & $\begin{array}{l}\text { VALOR DE } \\
\text { CAPTACIÓN (dólares } \\
\qquad / \mathrm{m}^{3}\end{array}$ & $\begin{array}{l}\text { Costo total por } \\
\text { (USD)año/ Ha }\end{array}$ \\
\hline Agropecuario & $6,739.20$ & 0.0565 & 0.000278 & \\
\hline
\end{tabular}

* Demanda de 52 semanas se supone un riego por semana.

Fuente: Elaboración Propia de los Autores

tauración se determina la importancia económica del servicio ambiental hídrico que es el siguiente:

Los valores planteados en la tabla 2, muestran que se debe empezar a destinar recursos para la conservación de los afluentes, para que en él futuro no disminuya el caudal del río Guargualla y por ende para el regadío de las comunidades que conforman el sistema de riego Guargualla Licto que beneficia a 2200 has de las 4 parroquias como son: San Pedro, San Miguel de Pungalá, Flores y Punan.

\section{MODELO ECONOMÉTRICO}

En base a los requerimientos del sistema y método para el cálculo de la valoración del recurso hídrico, es necesario seleccionar las variables correspondientes, siendo estas: características socioeconómicas, disponibilidad del recurso, diversidad de regiones y costo de oportunidad. Estas variables se integran en un modelo econométrico el cual se lo realizó en el programa SPSS. Para estimar el modelo econométrico se realizó una regresión lineal, se utilizó las variables más significativas para la disposición de pago y de esta manera se estimó según el modelo de la siguiente manera:

$$
\mathbf{Y}=\mathbf{a}+\mathbf{b} \mathbf{X} \mathbf{i}
$$

Donde:

$$
\begin{aligned}
& Y=\text { Disposición de Pago } \\
& a=\text { Intercepto } \\
& X i=\text { Variables correspondientes } \\
& \text { de las cuales corresponden a } \\
& \text { los cuatro factores } \\
& b i=\text { Coeficientes }
\end{aligned}
$$

Para estimar las probabilidades de que los beneficiarios estén dispuestos a pagar, se aplicó el estadístico Chi Cuadrado, el mismo que nos permitió descartar las va- 
Tabla 3. Coeficientes

\begin{tabular}{|c|c|c|c|c|c|c|c|}
\hline \multirow[b]{2}{*}{ Modelo } & \multicolumn{2}{|c|}{$\begin{array}{l}\text { Coeficientes no } \\
\text { estandarizados }\end{array}$} & \multirow{2}{*}{$\begin{array}{c}\text { Coeficientes } \\
\text { tipificados } \\
\text { Beta }\end{array}$} & \multirow[b]{2}{*}{$t$} & \multirow[b]{2}{*}{ Sig. } & \multicolumn{2}{|c|}{$\begin{array}{c}\text { Intervalo de } \\
\text { confianza de } 95,0 \% \\
\text { para B }\end{array}$} \\
\hline & B & Error típ. & & & & $\begin{array}{l}\text { Límite } \\
\text { inferior }\end{array}$ & $\begin{array}{l}\text { Límite } \\
\text { superior }\end{array}$ \\
\hline 1 (Constante) &,- 164 &, 462 & &,- 355 &, 723 & $-1,072$ &, 744 \\
\hline ¿Cuál es su edad? &,- 031 &, 008 &,- 364 & $-3,865$ &, 000 &,- 047 &,- 015 \\
\hline ¿Nivel de instrucción? &,- 030 & ,056 &,- 039 &,- 540 & ,589 &,- 140 &, 079 \\
\hline $\begin{array}{l}\text { ¿Número de personas que } \\
\text { viven en el hogar? }\end{array}$ & ,389 & ,056 & ,831 & 6,980 & ,000 & ,279 & , 498 \\
\hline $\begin{array}{l}\text { ¿Sus ingresos económicos } \\
\text { mensuales son? }\end{array}$ & ,379 & ,131 & ,177 & 2,884 & ,004 &, 120 & ,637 \\
\hline $\begin{array}{l}\text { ¿Cómo se abastece del } \\
\text { suministro del agua? }\end{array}$ & ,397 & ,208 & ,178 & 1,908 & ,057 &,- 013 & ,807 \\
\hline $\begin{array}{l}\text { ¿Ha notado en los últimos } 4 \\
\text { años que el suministro de } \\
\text { agua está disminuyendo? }\end{array}$ & ,185 & ,153 & ,075 & 1,210 & 227 &,- 116 & ,486 \\
\hline $\begin{array}{l}\text { ¿Cuál considera usted son } \\
\text { los factores de la escasez } \\
\text { del agua? }\end{array}$ & 197 & ,051 & ,206 & 3,897 & ,000 & ,097 & 296 \\
\hline $\begin{array}{l}\text { ¿Qué beneficios ha } \\
\text { ocasionado el riego en los } \\
\text { últimos } 4 \text { años? }\end{array}$ &,- 162 & ,063 &,- 129 & $-2,583$ &, 010 &,- 285 &,- 039 \\
\hline $\begin{array}{l}\text { ¿Estaría de acuerdo a pagar } \\
\text { por las acciones de } \\
\text { conservación de las fuentes } \\
\text { de aguas que usted utiliza? }\end{array}$ & ,474 & ,180 & ,130 & 2,625 & ,009 & ,119 & ,829 \\
\hline
\end{tabular}

Fuente: SPSS

riables a través del cálculo del nivel de significancia de cada una de las variables independientes en relación con la variable dependiente, de esta manera el modelo de estimación permitió conocer la disposición de pago de los beneficiarios.

La función de utilidad de los beneficiarios con base a las variables estudiadas

$$
\begin{gathered}
\text { DAP }_{i j}=f\left(\text { CARSOC }_{i j} ; \text { DISREC }_{i j}\right. \\
\text { DIVERG } \left._{i j} \operatorname{COSTOP~}_{i j}\right)
\end{gathered}
$$

El modelo estadístico que se plantea es el siguiente:

$$
\begin{aligned}
& \text { DAP }_{i j}=a+b\left(\text { CARSOC }_{i j}\right)+ \\
& c\left(\text { DISREC }_{i j}\right)+d\left(\text { DIVERG }_{i j}\right)+ \\
& j\left(\operatorname{COSTOP}_{i j}\right)+m_{i j}
\end{aligned}
$$

\section{Dónde:}

$D A P_{i j}=$ Disponibilidad a pagar por los beneficiarios del recurso.

$C_{C A R S O C}=$ Conjunto de las variables que definen las características socioeconómicas de los beneficiarios del sistema.

$D_{I S R E C_{i j}}=$ Conjunto de variables que definen la disponibilidad del recurso para el beneficiario en la fuente. 
Valoración económica ambiental del recurso hídrico..

DIVERG $G_{i j}=$ Conjunto de variables que definen la diversidad de la región donde el beneficiario capta el recurso.

COSTOP $i j=$ Conjunto de variables que definen el costo de oportunidad del recurso.

$m_{i j}=$ es el termino de error, distribuido independientemente por los beneficiarios.

De la investigación realizada podemos determinar que solo 9 de las variables tienen un alto nivel de significancia, las cuales permitieron la estimación del modelo econométrico. Una vez calculado el nivel de dependencia de las variables a través de Chi Cuadrado, se procedió a medir la correlación entre la variable dependiente con las variables independientes, para lo cual se utilizó el coeficiente de contingen- cia, considerando que éste expresa la intensidad entre dos o más variables.

Ya calculado el nivel de dependencia de las variables a través del Chi Cuadrado, se procede a medir la CORRELACIÓN entre la variable DEPENDIENTE con las variables INDEPENDIENTES, para lo cual se utilizó el coeficiente de contingencia considerando esta ya que expresa la intensidad entre dos o más variables. La fórmula del cálculo de Coeficiente de Contingencia es:

$$
\mathrm{C}=\sqrt{\frac{X 2}{X 2+n}}
$$

\section{Dónde:}

$C=$ Coeficiente de Contingencia X2 = Chi Cuadrado

$n$ = Tamaño de la muestra

\section{Tabla 4. Chi Cuadrado y Coeficiente de Contingencia}

\begin{tabular}{l|c|c|}
\hline \multicolumn{1}{|c}{ Variables } & Chi Cuadrado & $\begin{array}{c}\text { Coeficiente de } \\
\text { Contingencia }\end{array}$ \\
\hline Edad & 0.001 & 0.654 \\
\hline Instrucción & 0.000 & 0.436 \\
\hline Habitan & 0.000 & 0.717 \\
\hline Ingreso & 0.000 & 0.254 \\
\hline Suministro & 0.000 & 0.272 \\
\hline Reducción & 0.000 & 0.435 \\
Escasez & 0.000 & 0.464 \\
\hline Beneficio & 0.000 & 0.396 \\
Acciones & 0.000 & 0.355
\end{tabular}

Fuente: SPSS 
Luego de haber realizado el cálculo de coeficiente de contingencia, se realizó una regresión lineal, donde se utilizó las variables más significativas para la disposición de pago.

$$
Y=a+b X
$$

\section{Dónde:}

$$
\begin{aligned}
& Y=\text { Disposición de Pago } \\
& a=\text { intercepto } \\
& X_{i}=¿ \text { Estaría de acuerdo a pagar } \\
& \text { por las acciones de conserva- } \\
& \text { ción de las fuentes de aguas } \\
& \quad \text { que usted utiliza? } \\
& \mathrm{b}_{\mathrm{i}}=\text { Coeficientes }
\end{aligned}
$$

$$
\text { DAP }=-0,164+0,474(X)
$$

Si la variable $\mathrm{X}$ toma el valor de 1 , tendríamos una disposición de pago de $=0,31$

Según el resultado, el pago para la conservación del recurso hídrico es de 0,31 centavos de dólares por usuario. Lo que significa que debe incluir en sus pagos este valor para destinar al cuidado y conservación de los páramos y así disponer de agua para riego en las futuras generaciones.

\section{DISCUSIÓN}

La distribución de la renta en el caso ecuatoriano se ha llevado a cabo utilizando instrumentos de política asistencial, productiva y de desarrollo de infraestructuras públicas (puentes, hospitales, carreteras, hidroeléctricas y etc.). Esta particular distribución de la renta privilegió sensiblemente al gasto de inversión y muy poco a la generación de tecnología y de valor agregado en la agrícultura y casi nada en la dotación de sistemas de riego tecnificados.

En los últimos años el Ecuador se ha interesado en mejorar la distribución equitativa del agua, y de la misma forma invertir en mejorar los sistemas riego estatales y comunitarios lo que ha generado una mayor confianza para que beneficiarios inviertan en mejorar la calidad del producto.

\section{CONCLUSIONES}

- El riego ha generado ingresos económicos al $92 \%$ de las familias, el $36 \%$ de los usuarios cuentan con un ingreso económico mensual por hectárea de 100 USD dólares, el $29 \%$ igual 
Valoración económica ambiental del recurso hídrico..

a 375 dólares, el $6 \%$ entre el rango de 375 a 500 , el $3 \%$ entre el rango de 501 a 800 , el $3 \%$ entre el rango de 801 a 1200 dólares. para solventar sus necesidades básicas.

- El estudio estableció que el $82 \%$ de los usuarios están conscientes de la disminución del recurso hídrico, por lo que el $91 \%$ están dispuestos a contribuir con un pago, para la conservación de los páramos.

- Por la metodología de contingente, se determina que el valor de restauración es de 0.0002781876 por $\mathrm{m} 3$, y el valor de captación es de 0.0564809624 centavos de dólar por m3.

- El valor de pago para la conservación del recurso hídrico 0,31 centavos de dólar por usuario, que permitirá financiar la conservación de las fuentes del sistema de riego de la Junta Guargualla.

\section{BIBLIOGRAFÍA}

Azqueta. (2002). Valoración económica del medio ambiente. Madrid: Editorial Mc. Graw Hill.

Cerda, A. (2009). Valoración Económica del Ambiente. Obtenido de https://www.cepal.org/ilpes/noticias/paginas/8/35988/ivaloracioncepal2009.pdf
Herruzo. (2002). Fundamentos y métodos para la valoración de bienes ambientales.

Organización de las Naciones Unidas. (2016). Informe de las Naciones Unidas sobre el Desarrollo de los Recursos Hídricos en el Mundo.

Obtenido de Agua para todos agua para la vida:

http://www.un.org/esa/sustdev/sdissues/water/WWDR-spanish129556s.pdf

Riera, P. (1994). Manual de valoración contigente. Distrito Federal de México.

Romero, C. (2014). Economia de los recursos ambientales y naturales. Madrid: $2^{\mathrm{a}}$ edición, Alianza Editorial.

Tomasini, D. (2018). Valoración Económica del Ambiente. Obtenido de http://www.keneamazon.net/Documents/Publications/Virtual-Library/Economia-Desarrollo/122.pdf 